\title{
LA ORGANIZACIÓN POLÍTICA Y EL PAISAJE DE CHICHÉN ITZÁ, YUCATÁN, MÉXICO, EN EL PERÍODO CLÁSICO TERMINAL (830-930 dC)
}

\author{
Péter Bíró y Eduardo Pérez de Heredia
}

\begin{abstract}
Este artículo examina la organización política de la ciudad prehispánica de Chichén Itzá, Yucatán, México, durante el reinado de K'ak' Upakal K'inich K'awil en la segunda mitad del siglo IX dC. Las interpretaciones políticas y territoriales que presentamos se basan en un entramado cronológico en el que entendemos los sectores del Chichén Itzá "Maya" y el Chichén Itzá "Tolteca" como dos periodos diferentes y secuenciales. Examinamos los cambios que aparecen en el período Clásico terminal, tales como las nuevas características no Mayas, el uso de glifos y nombres de origen foráneo, así como dioses de las Tierras Altas de México y nuevos tipos de edificios. En esta contribución también mostramos cómo el sistema interno de organización social cambió de un modelo de monarquía de un rey absoluto, identificado con la divinidad, a un tipo de gobierno más estructurado e incluyente, con mayor poder de la nobleza. Finalmente, proponemos un patrón de asentamiento que refleja esta nueva organización sociopolítica.
\end{abstract}

We examine the political organization of the prehispanic city of Chichen Itza, Yucatan, during the reign of K'ak' Upakal $K$ 'inich K'awil in the second half of the 9th century $A D$. The political and territorial interpretations we present are based on a chronological framework where "Maya" and "Toltec" Chichen Itza date to two different, sequential periods. We examine the changes that appear in the Terminal Classic including the introduction of new non-Maya characteristics such as foreign glyphs, central highland gods, and new types of public buildings. We also demonstrate how political organization changed from the one-king model of the Classic Maya, in which the ruler identified himself with the gods, to a more structured, ranked type of government that required the inclusion of more nobility. Finally, we propose that settlement patterns reflect this new sociopolitical organization.

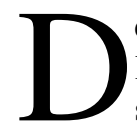
esde el desciframiento del nombre de K'ak' Upakal por David Kelley en 1968, se ha avanzado considerablemente en el entendimiento de la ciudad prehipánica de Chichén Itzá y la vida de este gobernante Maya, revelando un período de importantes cambios en la historia de esta cultura (Pérez de Heredia y Bíró 2017; Pérez de Heredia y Bíró 2018). El mismo corresponde al período Clásico terminal (siglo noveno dC), el cual fue un breve período de intensa actividad constructiva en Chichén Itzá. En un lapso de tiempo relativamente corto fueron construidos varios edificios monumentales concentrados en las cercanías del Cenote Xtolok (Figura 1). Muchas de estas construcciones cuentan con inscripciones con fechas inaugurales, las cuales son los rasgos más fiables en que basar la cronología de la ciudad, y que se concentran entre 860 y $890 \mathrm{dC}$.

En términos de entender la organización política de Chichén Itzá, la propia cronología está en debate. La mayoría de los investigadores que abordan este tema, en las últimas tres décadas, ha operado bajo los modelos del 'traslape total' o del 'traslape parcial' (Sabloff y Andrews 1986), los cuales consideran los sectores arquitectónicos "Maya" y "Tolteca"1 de Chichén Itzá como total o parcialmente contemporáneos. Por ello debieron proveer un modelo de organización social que fuera capaz de explicar ambos fenómenos sincrónicamente, encontrando muchos problemas y contradicciones en este esfuerzo.

En cuanto a la cerámica de Chichen Itzá, en este trabajo seguimos un modelo cronológico

Péter Bíró — Historiador independiente, Tiszavasvári, Hungría (bpetr30@ gmail.com)

Eduardo Pérez de Heredia — Historiador independiente, Mérida, Yucatán, México (kakupakal@ gmail.com)

Latin American Antiquity 29(2), 2018, pp. 207-221

Copyright (C) 2018 by the Society for American Archaeology doi:10.1017/laq.2017.85 


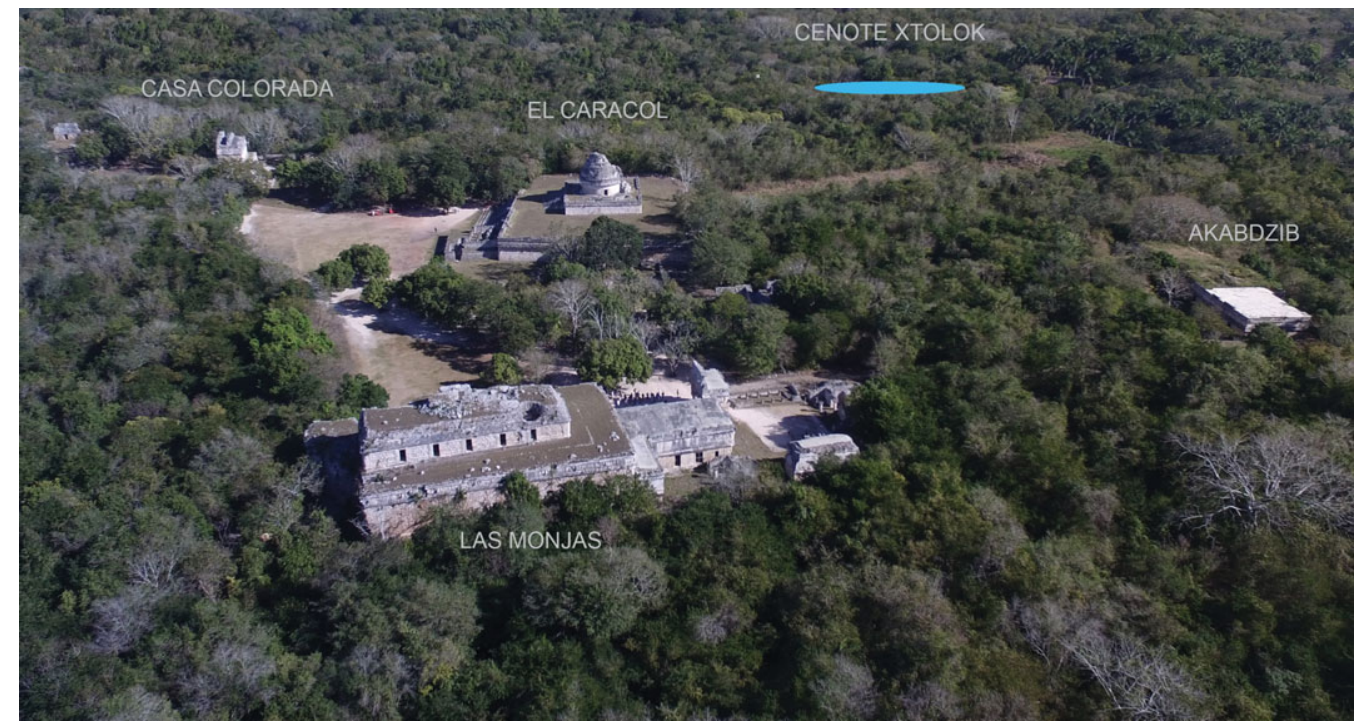

Figura 1. Edificios principales y distribución del centro de Chichen Itzá en el Clásico terminal (fotografía por Eduardo Pérez de Heredia). (Color online)

de no traslape, como hicieron George Brainerd (1958) y Robert E. Smith (1971) anteriormente (con modificaciones en las fechas de inicio y fin de los complejos cerámicos Cehpech y Sotuta). Según este modelo tradicional, las construcciones asociadas "Mayas" y "Toltecas" son consideradas fenómenos secuenciales $\mathrm{y}$, en consecuencia, la organización política ha de explicarse por separado (Pérez de Heredia 2010; ver críticas a esta cronología en Johnson [2015], Ringle [2017] y Volta y Braswell [2014]). En este artículo nos concentramos en las construcciones "Mayas" del periodo Clásico terminal, correspondiente al siglo noveno dC. Una datación del inicio de la ciudad "Maya" de Chichén Itzá ligeramente después de 800 dC está soportada no solamente por el registro epigráfico (Boot 2005; Thompson 1937) sino también por los análisis recientes de la evidencia cerámica (Pérez de Heredia 2010).

Procederemos primero a revisar los modelos existentes de interpretación de la organización política de Chichén Itzá, con especial énfasis en los diferentes enfoques de interpretación, ya sean modelos de gobernante único o de más de un gobernante. Después presentaremos nuestra nueva interpretación, en la cual proponemos que el sistema de organización política cambió de un modelo de gobernante único del período Clásico
Maya a un modelo más complejo en que el gobernante compartía autoridad con los miembros de la familia real y con la nobleza a través de dos grupos tríadicos, ambos comandados por K'ak' Upakal. Adicionalmente, mostraremos como estos grupos políticos principales se dividieron la ciudad y el territorio circundante, seleccionando una localización de los grupos arquitectónicos en posiciones privilegiadas entre rejolladas, el tipo de tierra más valioso en las tierras bajas del norte dado que sirven de huertas para especies altamente apreciadas como el cacao. El Cenote Xtolok sirvió como eje de la configuración del nuevo desarrollo urbano proveyendo de una fuente permanente de agua, a la par de estar posiblemente sacralizado, como sugiere la cercana localización del edificio del Caracol, al cual podría haber estado unido ya desde esta época por un sacbe (calzada).

\section{La Organización Política de Chichén Itzá: Interpretaciones Previas}

Un problema recurrente para los estudiosos de la cultura Maya estriba en que las primeras fuentes españolas acerca de la historia del norte de Yucatán son muy confusas, cuando no abiertamente contradictorias. Esto es especialmente grave en lo que respecta a la organización 
política de Chichén Itzá (Boot 2005). Por un lado, el obispo Diego de Landa menciona dos importantes participantes: el grupo Itzá y la persona histórica K'uk'ulkan, pero la relación entre ambos no era clara ni para el mismo fraile en aquel entonces. En otra ocasión se refiere a tres hermanos-sacerdotes que gobernaron simultáneamente en Chichén Itzá. Del mismo modo, las Relaciones Geográficas de Yucatán se refieren a un rey que gobernó Chichén Itzá, controlando desde allí una vasta área del sur de Mesoamérica (De la Garza et al. 1983).

Por otro lado, las fuentes coloniales nativas (como los Chilam Balam) contienen muchas referencias a los Itzá, y menos menciones de K'uk'ulkan. En cualquier caso, el género de los ciclos de $k$ 'atunes es difícil de ajustar consistentemente a los diferentes entramados arqueológicos, lo cual da lugar a diferentes cronologías que abarcan de la versión larga a la corta (Barrera Vázquez y Rendón 1948; Boot 2005; Edmonson 1982, 1986; Roys 1962; Schele y Mathews 1998).

Desde los años 1930 hasta los 1970, la mayoría de los estudiosos coincidían en una interpretación general del auge y caída de Chichén Itzá con un periodo Maya y otro Tolteca (Morley 1946; Thompson 1954; Tozzer 1957). Sin embargo, en estas reconstrucciones la organización política del sitio nunca fue detallada, limitándose a describirla como una capital del Nuevo Imperio, o una capital del Imperio Tolteca.

Durante los años 1970 a 1990, datos de otros sitios arqueológicos del norte de Yucatán produjeron cambios en la cronología, con la presentación de modelos alternativos de secuencias cerámicas en los cuales se aceptaba una parcial o total contemporaneidad de los sectores "Maya" y "Tolteca" de la ciudad (e.g., Andrews y Robles 1985; Andrews, Andrews V y Robles 2003; Andrews V y Sabloff 1986; Kristan-Graham y Kowalski 2007). Bajo este nuevo escenario de contemporaneidad, diversos estudiosos construyeron ideas acerca de la organización política del sitio (Boot 2005:30; Kowalski y KristanGraham 2007; Lincoln 1990; Ringle 2004, 2009; Ringle et al. 1998:188-192; Schele y Freidel 1990:355; Schele y Mathews 1998:199-200; Wren y Schmidt 1991). En ellas Chichén Itzá se percibía como una de las ciudades tipo del período Epiclásico, a la manera de Cacaxtla, Xochicalco, o El Tajín.

No obstante, la mecánica de la organización sociopolítica y los detalles históricos continuaron siendo motivo de debate (Baudez y Latsanopoulos 2010; Boot 2005; Davoust 1977; Grube 1994, 2003; Grube y Krochok 2007; Ringle 2004, 2009; Ringle et al. 1998; Schele y Freidel 1990:346-376; Schele y Mathews 1998:19725). En este sentido, existía un desacuerdo en cuanto a la organización política, con algunos investigadores soportando la idea de un gobernante supremo, mientras otros proponían el gobierno de más de un rey simultáneamente. A modo de ejemplo se puede mencionar a Linda Schele y David Freidel (1990:348) quienes reconstruyeron tres grupos de hermanos (o familias) sugiriendo que ellos controlaron la ciudad entre 800 y $1000 \mathrm{dC}$ (ver también Schele y Mathews [1998:198-199]). Aunque esta idea gozó de cierta popularidad en su momento, poco después otros epigrafistas determinaron que muchos de los miembros de esos grupos propuestos no eran personajes históricos sino dioses, reduciendo significativamente el número de los actores involucrados en la política de Chichén Itzá.

Por su parte, Alexander Voss y Jürgen Kremer (2000) propusieron que en el siglo noveno dC Chichén Itzá dominó a Yula, Halakal y Ek Balam, estando gobernada por un triunvirato que incluía tres títulos diferentes: el Capitán Disco Solar, el Capitán Serpiente y el Kokom. Propusieron que el Capitán Disco Solar evolucionó después hacia el Ah Canul mientras el k'uhul kokom se convirtió en un oficio-patronímico en Mayapán (Voss y Kremer 2000:169). Asimismo, consideraron la posibilidad de que estos tres señores fuesen los mismos que los hermanos-sacerdotes mencionados por Landa (Voss y Kremer 2000: 171).

Diversos estudiosos han propuesto que existió un sistema dual de gobierno en Chichén Itzá, donde el primer gobernante mantendría mayor autoridad que el segundo. William Ringle (2004:213) ha sugerido que un líder central gobernaba Chichén Itzá a través de un consejo, donde el líder supremo tendría la supremacía como capitán de guerra. Por el contrario, Eric Boot (2005) argumentó que el concepto de 
multepal (gobierno compartido) no existió en Chichén Itzá, proponiendo en cambio que el gobernante supremo era K'ak' Upakal y su oficio (k'uhul ajaw) estaba simbolizado por el "Capitan Disco Solar”, mientras su hermano K'inil Kopol habría tenido un oficio religioso. Este autor comparó este caso con un par de gobernantes de los Itzá del período colonial del Lago Petén Itzá, advirtiendo finalmente que, aunque K'ak' Upakal fue posiblemente el gobernante supremo, no existe evidencia explícita de ello (tal como la fórmula de acceso de otros textos del Clásico; Boot 2005:428). Más recientemente, Nikolai Grube y Ruth Krochock han notado que, aparte de K'ak' Upakal, su hermano también gobernó en la ciudad, aunque en un rango menor, admitiendo que no existen en los textos "indicaciones claras de la jerarquía entre ambos hermanos" (2007:240).

\section{Los Textos Jeroglíficos y la Política de Chichén Itzá en el Siglo Noveno: Los Títulos}

Definitivamente la persona más importante en el gobierno, por el número de referencias y por la calidad de sus títulos, fue K'ak' Upakal K'inich K'awil. Con la sola excepción del Akabdzib, todos los edificios con inscripciones entre 869 y 890 dC en Chichén Itzá y sitios circundantes se refieren a K' ak' Upakal en una posición principal en el discurso (Plank 2003). La segunda persona en número de menciones es Uchok Waj Uta'ab', muy posiblemente el padre de K'ak' Upakal, y quien fue registrado en los edificios alrededor del Templo de los Tres Dinteles (Cuadrante 7B del mapa de Carnegie [Ruppert 1952]), el Grupo de la Serie Inicial, la Casa Colorada y el Caracol (Figura 1), aunque nunca en una posición de agente. La tercera persona más mencionada es K'inil Kopol, hermano de K'ak' Upakal, como sugirió primero Ruth Krochock (1988), apareciendo en el mismo Cuadrante 7B y en el Grupo de la Serie Inicial. En el cuarto lugar se encuentra Ak'e Tok', también con menciones en el Cuadrante 7B y en el grupo de Serie Inicial, y quien comparte el glifo yitaj con K'inil Kopol. Otro importante personaje, el k'uhul kokom (que podría traducirse como divino juez) ocurre en el Akabdzib, la Casa Colorada y el Dintel del Bebedero. Finalmente, el título k'uhul aj kan (divino intérprete) está confinado al complejo del Caracol.

Shannon Plank (2003:397) fue la primera en notar que K'ak' Upakal, Uchok Waj Uta'ab', K'inil Kopol y el k'uhul kokom nunca usaron entre ellos el termino yitaj (él/ella acompaño a él/ella/ello; ver Macleod [2004]). De hecho, esta situación es aplicable a una quinta persona, $\mathrm{Aj}$ B'olon K'awil, quien presumiblemente tenía el control del complejo del Caracol. En el discurso del Clásico Maya los humanos reciben múltiples epítetos, usualmente en parejas semánticas (Carrasco y Hull 2014). Por ejemplo, en el Templo de los Cuatro Dinteles K'ak' Upakal es mencionado como $k^{\prime}$ uhul aj mel waj (divino que distribuye tamales [Dmitri Belaev, comunicación personal 2014]) y k'uhul waj ujaw, mientras ox pakab' matan (tres que pagan las caridades recibidas [Voss y Kremer 2000:172-173]) fue un título aplicado solamente a tres personas del grupo: K'ak' Upakal, K'inil Kopol y Ak'e Tok'.

Plank (2003:397) también reconoció que el padre común de K'ak' Upakal y K'inil Kopol fue Uchok Waj Uta'ab, quien, de acuerdo a esta investigadora, ya habría fallecido cuando fueron realizadas las inscripciones de K'ak' Upakal, como puede interpretarse de los textos de Monjas, Tres Dinteles y Un Dintel. De la lista de nombres y su localización se desprende también que la familia de K'ak' Upakal era la propietaria de la mayoría de los edificios con inscripciones en la segunda mitad del siglo noveno, incluyendo las Monjas, el grupo de la Casa Colorada, los edificios del Cuadrante 7B y el Grupo de la Serie Inicial. Aparte de ellos, el Akabdzib pertenecía a la familia del k'uhul kokom y el Caracol quedaba bajo el control de Aj B'olon K'awil, el k'uhul aj kan.

Dos triadas pueden ser inferidas de los datos mencionados arriba, ambas lideradas por K'ak' Upakal:

Tabla 1. Dos triadas de Chichen Itzá ente 869 y 890 dC.

\begin{tabular}{ll}
\hline Área Sur & Área Norte \\
\hline K'ak' Upakal & K'ak' Upakal \\
K'inil Kopol & K'uhul Kokom \\
Ak'e Tok' & K'uhul Aj Kan \\
\hline
\end{tabular}



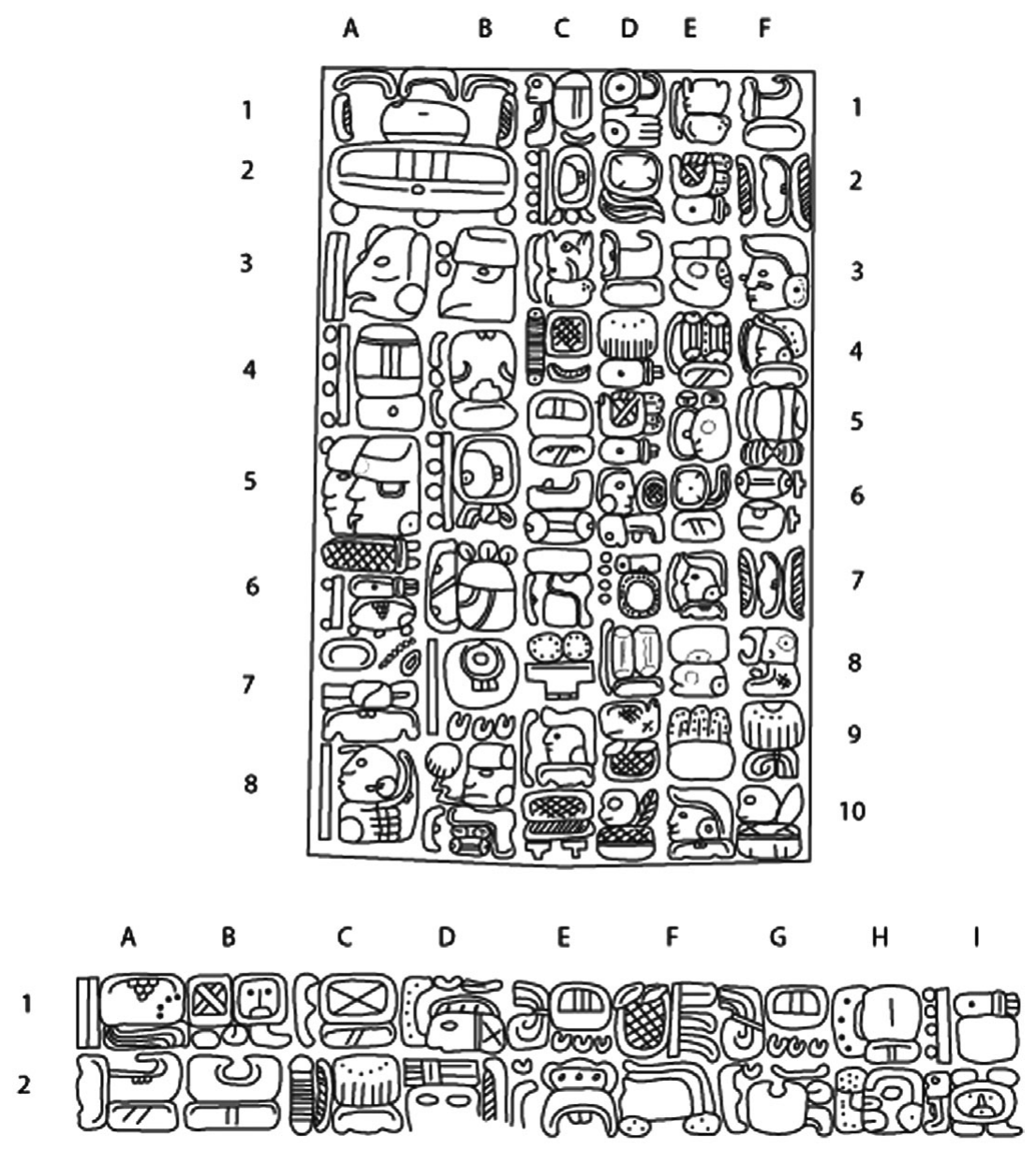

Figura 2. Dintel de la Serie Inicial, o Casa de los Cuatro Braseros (dibujo de Ruth Krochock [1989:8, fig.1], modificado por Péter Bíró).

La primera triada residía en la parte sur de la ciudad y reclamaba su legitimidad a través de la figura de Uchok Waj Uta'ab', como está descrito explícitamente en el Dintel de la Serie Inicial (Figura 2). Este texto, dedicado en 878 dC, narra la animación de la casa de Uchok Waj Uta'ab', refiriéndose a la construcción como la Casa de los Cuatro Braseros. En el texto existe un difrasismo (el pareado de distintos elementos para producir un tercer concepto metafórico, más abstracto) compuesto de la palabra kok "guardar" y el indescifrado título PTB del catálogo de Macri y Looper (Figura 3a: Grube, Lacadena y Martin 2003:ii-51). Este grafema PTB es único en las inscripciones de las tierras bajas, aunque aparece como motivo iconográfico en la columna de la Estructura 6E1 (Figura 3b), donde tres de los cuatro personajes presentan la peculiar nariguera y uno de ellos muestra el corte de cabello temotlli, que también aparece en Seibal y Machaquila, y posteriormente devendría en el signo de tecuhtli en el Postclásico del Centro de México.

Por la sintaxis puede entenderse que el PTB era guardado primero por K' ak' Upakal, después por K'inil Kopol y finalmente por Ak'e Tok'. Un patrón muy interesante es que la primera cláusula lista la casa del padre y de K'ak' Upakal, y la segunda cláusula menciona la casa del kanak te' (segunda lanza) PTB K'inil Kopol. Este patrón aparece también en el Templo de los Cuatro Dinteles, donde los mismos señores - K'ak' Upakal, K'inil Kopol y Ak'e Tok' - aparecen enlistados en orden, el último siempre con la expresión yitaj. De hecho, Ak'e Tok' nunca 

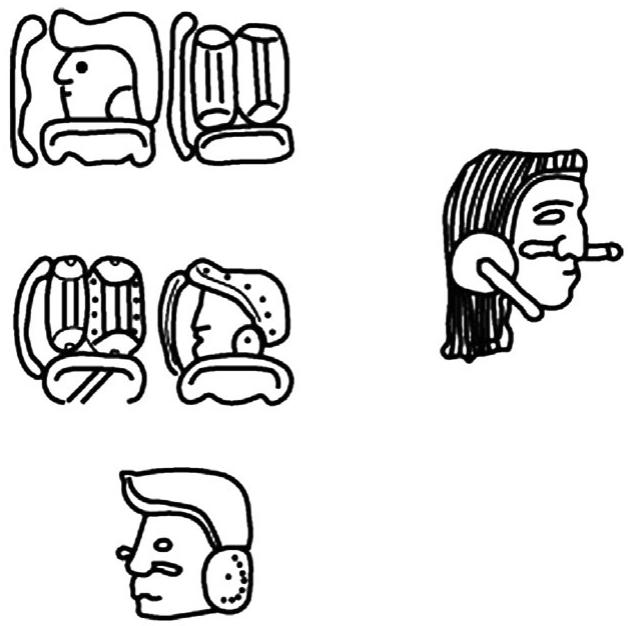

a

b

Figura 3. Ejemplos del compuesto de $k o k$ y el grafema de PTB en el registro epigráfico de Chichén Itzá: (a) bloques D8-C9, E4-F4 y el PTB sólo en F3 de la inscripción del Dintel de la Serie Inicial; (b) detalle de la Columna 6E1, el corte de cabello temotlli (dibujo de Linda Schele [Schele y Freidel 1990:361, fig. 9:13], modificado por Péter Bíró).

aparece actuando solo, mientras K'ak' Upakal y K'inil Kopol condujeron rituales por sí mismos. Por ejemplo, K'inil Kopol realizó el ritual de Fuego de los dinteles 2 y 3 del Templo de los Cuatro Dinteles.

La segunda triada ocupaba el sector norte, donde el k'uhul kokom residía en el noreste (Akabdzib), el k'uhul aj kan ocupaba el Caracol (Templo de Quetzalcoatl) y K'ak' Upakal controlaba la parte noroeste (Monjas-Casa Colorada). Podemos encontrar un patrón de tríos de actores en numerosos textos de Chichén Itzá:

Tabla 2. Patrón entre los textos y nombres de Chichén Itzá.

\begin{tabular}{lc}
\hline Inscripciones & Nombres \\
\hline Casa Colorada & K'ak' Upakal, K'uhul Tzol Ajaw (Ek \\
& Balam), K'uhul Kokom \\
Halakal Dintel & K'uhul Tzol Ajaw, K'ak' Upakal, ? \\
& Ajaw \\
Yula Lin. 1 \& 2 & K'ak' Upakal, K'uhul Um, Koj Ajaw \\
Initial S. & K'ak' Upakal, K'inil Kopol, Ak'e Tok' \\
TL4 Lin 1 & K'ak' Upakal, K'inil Kopol, Ak'e Tok' \\
TL4 Lin 4 & K'ak' Upakal, K'inil Kopol, Ak'e Tok' \\
TL4 Lin 2 & K'inil Kopol, Ak'e Tok', K'uhul Kalaj \\
& Ukaw \\
Caracol Panel & K'ak' Upakal, anab'de Itzámal?, K'uhul \\
& Aj Kan \\
\hline
\end{tabular}

Apenas en cuatro ejemplos el ritual fue ejecutado por una sola persona: K'ak' Upakal en Monjas, K'inil Kopol en el Templo de los Cuatro Dinteles y k'uhul kokom en el Dintel del Bebedero y el Akabdzib, y solo en una instancia K'ak' Upakal y K'inil Kopol son mencionados juntos sin otra compañía (Dintel 1 de Tres Dinteles). Otro patrón de interés que puede discernirse es que todos los actores importantes de Chichén Itzá tienen al menos un título k'uhul, un caso único en las inscripciones de las tierras bajas.

Tabla 3. Patrón entre los nombres y los títulos con $k^{\prime} u h u l$.

\begin{tabular}{ll}
\hline Nombres & \multicolumn{1}{c}{ K'uhul títulos } \\
\hline K'ak' Upakal & $\begin{array}{c}\text { k'uhul ajaw, k'uhul aj mel waj, } \\
\text { k'uhul aj k'ahk', k'uhul waj } \\
\text { ujaw } \\
\text { k'uhul aj mel waj, k'uhul yajaw } \\
\text { k'ahk' } \\
\text { K'inil Kopol }\end{array}$ \\
A'uhul waj ujaw \\
Ak'e Tok' & k'uhul kokom \\
Yajaw Choh? & k'uhul aj kan \\
Aj B'olon K'awil & k'uhul um \\
Tok' Yas Ajaw & k'uhul tun \\
Kalkej Tok Puj Ajaw & k'uhul kalaj ukaw \\
Aj ? &
\end{tabular}

El análisis de las relaciones de parentesco presentes en los textos nos muestra dos parejas de hermanos, la primera formada por K'ak' Upakal y K'inil Kopol y la segunda por Tok' Yas Ajaw y Kalkej Tok Puj Ajaw en Yula. En relación al primer par, Mark Zender (2004:245246) ha notado que en la Relación de Diego de Landa el segundo de los hijos era consignado al sacerdocio. Aparte del título k'uhul aj mel way, K'inil Kopol era k'uhul yajaw k'ahk' (divino señor-vasallo del fuego), lo que lo coloca en el primer lugar del escalafón sacerdotal. Aunque tanto Ak'e Tok' como Aj ? ostentan el título de yajaw k'ahk', el adjetivo k'uhul señala que K'inil Kopol se encontraba en la cima de la jerarquía de los Señores del Fuego, únicamente segundo detrás de K'ak' Upakal.

Los miembros de cada familia eran dueños de diferentes dioses o ancestros deificados. Por ejemplo, la familia de K'ak' Upakal poseía al dios más importante, que era Yax Pech Kan (Verde Pato Serpiente), identificado con el dios Ehecatl (aspecto de Quetzalcoatl), cuyos propietarios eran K'ak' Upakal, K'inil Kopol y Ak'e 
Tok', "los tres que pagan las caridades recibidas". Por su parte K'inil Kopol poseía a Uchok Yok Puy, mientras el dios Yax K'uk'um K'awil pertenecía al mismo K'ak' Upakal. Otro caso era el de los Kokomes, que eran dueños de un dios diferente (registrado en el Dintel del Bebedero). En Yula el dios patrón era posiblemente Yax Ha'al Chahk.

En resumen, los textos de Chichén Itzá durante el Clásico terminal presentan patrones que permiten esbozar un tipo de organización política que representa una novedad en las tierras bajas mayas. Aunque solamente existía un $k^{\prime}$ 'uhul ajaw (divino señor, o gobernante supremo del período Clásico) en la persona de K'ak' Upakal, existían dos tríos de personas que tenían diferentes roles en el gobierno de la ciudad. Esta organización política evolucionó al parecer desde el sistema de k'uhul ajaw de las tierras bajas del sur, especialmente de la región oeste, donde ya en el período Clásico temprano el discurso enfatizaba los logros e importancia de los miembros de la nobleza, y donde yajaw $k$ 'ahk' (Señor del Fuego) era un título prominente (Bíró 2011).

De cualquier modo, el desarrollo más importante es que la nobleza comparte el adjetivo $k^{\prime} u h u l$, una evolución única en las tierras bajas que podría estar conectada con la región oeste, donde en Toniná un noble usa el título k'uhul ti' sak hun. Los datos examinados permiten pensar que, en este nuevo sistema, y al contrario que en el período Clásico, el gobernante supremo compartía efectivamente el poder con otros nobles, los cuales elevaban de esta manera su influencia y responsabilidad en la administración pública.

\section{Textos Coloniales}

No existe una descripción detallada del sistema de gobierno de Mayapán en las fuentes coloniales del norte de Yucatán, excepto menciones contradictorias del papel preponderante y la rivalidad de los linajes Kokom y Xiu, y la mención de un patrón territorial cuatripartito. La fuente directamente relacionada con Chichén Itzá proviene del Lago Petén Itzá, en las tierras bajas del sur, donde los Itzá se asentaron en tiempos del Postclásico tardío. Del estudio de estos documentos Grant Jones entiende que "un gobierno compartido era atemperado por un fuerte principio de dominación del linaje por un grupo de hombres cercanamente relacionados, dos de los cuales 'gobernaban' como una persona dual” (Jones 1998:60-107, nuestra traducción). El primero es el Ajaw Kan Ek' y el segundo es el Gran Sacerdote Aj K'in Kan Ek', quienes al tiempo de la crónica eran primos (Jones 1998:105-106). El dominio de los Itzá en el área lacustre del Petén tenía cuatro distritos, donde de nuevo aparece un par de oficiales (sénior-junior) para el gobierno de cada sector. Existían además otros consejeros con diferentes títulos, a veces difíciles de separar entre sí. Avendaño listó en 1696 al menos veintidós líderes entre los Itzá, cuatro de los cuales venían de la familia Kan Ek' (el gobernante anterior más tres hermanos [Jones 1998:84-87]). Es muy interesante comparar esta mención al gobernante fallecido y sus tres hijos con el Dintel de la Serie Inicial de Chichén Itzá, donde sucede lo mismo.

En las tierras altas de Guatemala, Robert Carmack $(1973,1981)$ detalla que en el dominio de los Quiché existían dos grupos de cuatro individuos: el primero es el aj pop, seguido de aj pop c'amja, nima rajpop achij y ch'uti rajpop achij; el segundo grupo está formado por el ajpop nuevamente, seguido del aj pop c'amja, k'alel y por último el atzij winak (Carmack 1981:169171). Mientras el primer grupo está integrado solamente por miembros del linaje Cawek, en el segundo los señores son miembros de tres linajes diferentes (Cawek, Nijaib, y Ajaw Quiché). El ajpop era el gobernante supremo en la capital y usualmente era sucedido por su hijo, cuyo título hasta su ascensión al poder era nima rajpop achij. En el otro grupo se encontraban los asistentes del rey para el gobierno efectivo del reino. El aj pop c'amja recibía a los visitantes, el k'alel era el juez principal y consejero y por último el atzij winak's (el vocero, o interprete) completaba la terna.

Estos nobles residían en complejos de varias casas grandes ( nim ja) controlando los precintos de templos y plazas. De acuerdo a algunos estudios la ciudad se hallaba dividida en cuatro sectores. En Utatlán existe una plaza principal con tres templos, uno de los cuales era poseído por los Cawek, y otro por los Nijaib, y entre 
ambas estructuras se localizaba el templo de Quetzalcoatl. Los Cawek, quienes usaban el título de mayor rango, residían en el oeste y parte del sur, y controlaban también el juego de pelota. El ajaw mismo ocupaba la otra parte del sur. Por su parte los Nijaib ocupaban el sector oriental y ostentaban el título k'alel (juez), y los Sakic, de menor rango, controlaban el norte (Carmack 1981:220-231; Fox 1989:91). Cada familia poseía también diferentes dioses. Como veremos más adelante, un patrón parecido de división territorial puede discernirse en el Chichén Itzá del siglo noveno.

En suma, los ejemplos mencionados arriba enfatizan el modelo de una regla general de división territorial cuadripartita. Aunque existen diferencias observables entre los diferentes modelos, en todos aparece una escala de rango dual de las oficinas de gobierno (sénior-junior). Aunque ciertos patrones estructurales pueden discernirse del discurso jeroglífico de Chichén Itzá, el contenido se enfoca en el aspecto ritual y no es muy informativo explícitamente acerca de la organización política. Sabemos que K'ak' Upakal fue invitado a otras partes del sitio para legitimar rituales. En Yula, él llegó (uliy) a la ciudad, y también fue invitado (pehkaj) al Templo del Caracol. También visitó oficialmente edificios en el Grupo de Tres Dinteles para cooficiar junto a su(s) hermano(s) y en Halakal recibió al rey de Ek Balam. Los textos de Chichén Itzá pueden verse desde esta perspectiva como un registro de las procesiones ceremoniales del gobernante supremo a las diferentes particiones (o sectores) de la ciudad y otros asentamientos vecinos, de manera similar a las ceremonias de fin de período de los gobernantes de Dos Pilas y otros reyes de las tierras bajas mayas en el Clásico tardío.

Sin embargo, en contraste con las tierras bajas del sur donde siempre se usaban expresiones claras para describir los eslabones jerárquicos entre las personas con título (por ejemplo, yajaw, usajal, yajk'uhun, uti'hun, etc.), en Chichén Itzá no se describía esta conexión particular y el orden jerárquico debe deducirse del orden de aparición en el discurso y la frecuencia de las menciones. La única excepción seria el mensajero foráneo mencionado en los textos del Caracol y que provenía posiblemente de Izamal, el cual usa el título yanab'il, que es una expresión jerárquica tradicional en el sistema de las tierras bajas del sur.

Esta organización política dual resuelve simultáneamente la línea de sucesión a la realeza mediante una de las triadas, donde todos los miembros son hombres cercanamente relacionados del linaje Kanek'/Cawek (el título de K'ak' Upakal), mientras el funcionamiento del gobierno se aseguraba con un trío de oficinas especializadas (militar, justicia y religión), asignadas a tres grupos diferentes. Las semejanzas del patrón mostrado para Chichen Itza con los ejemplos examinados del área Maya son mayores que si lo comparamos con ejemplos de las tierras altas del Centro de México, donde en algunos casos un altepetl tenía tres o más tlatoani, o aparecen combinaciones como la de Tenochtitlan, con la pareja de huei tlatoani y cihuacoatl (Kirchhoff 1955; Paulinyi 1981). La organización política de Chichén Itzá en el siglo noveno dC anticipa el sistema político Maya del período Postclásico tardío, en un ejemplo temprano de evolución desde el previo sistema del período Clásico. Este experimento fue truncado poco después de la muerte de K'ak' Upakal, con la llegada de personas no Mayas de origen mexicano a inicios del siglo décimo dC (Bíró y Pérez de Heredia 2016) que instauraron un nuevo orden en la ciudad, de mucha mayor complejidad.

\section{La Organización del Paisaje}

Varios autores han resaltado la relación de la ciudad con los más de una docena de cenotes en la vecindad como un factor primario para la ocupación, así como la presencia de depresiones kársticas conocidas como rejolladas (Gómez Pompa 1990; Kepecs y Boucher 1996; Schmidt 2003). En este sentido, parte medular del Proyecto Arqueológico Chichén Itzá INAH ha sido el estudio de las fuentes de agua del sitio, incluyendo rejolladas y las cisternas artificiales conocidas como chultunes (González de la Mata 2006; Schmidt 2007).

Varios intentos han sido realizados en las últimas décadas para discernir la organización sociopolítica de Chichén Itzá a través del análisis de su organización espacial. Charles Lincoln 
(1990) y Rafael Cobos (2004) han propuesto diferentes explicaciones para el arreglo arquitectónico en general y su relación con el sistema de calzadas de la ciudad, el primero de una manera sincrónica (es decir, como si toda la ciudad fuese contemporánea) mientras el segundo ha probado diferentes arreglos cronológicos. Si bien estos esfuerzos han contribuido a enriquecer el conocimiento del plano de la ciudad, no han logrado la formulación de un patrón concreto que explique comprehensivamente la configuración urbana de Chichén Itzá.

No se conoce un glifo emblema para el sitio, lo cual es significativo en sí mismo, pero en los textos de Chichén Itzá aparecen al menos dos nombres de lugar que quizás refieran a alguna de las aglomeraciones de edificios o grupos (aunque ninguno contiene el termino $c h$ 'en). En el primer caso, nosotros consideramos muy posible que el topónimo Tzaj o Tza' fuese el nombre del área dominada por la Torre del Caracol. El nombre aparece en textos del Caracol asociado con el noble Aj B'olon Kawil, quien era un "divino vocero-interprete" (k'uhul aj kan) y ostentaba el título "el del fuego", siendo la expresión completa (ajk'ahk') de Tzaj ... Tz'iknal (en) Wak(hab'?)nal (Panel del Caracol N1-N4; Serpiente Jeroglífica del Caracol Bloques 7, 8 y10 [Boot 2005:354; Voss 2001]).

Este texto del Caracol contiene también una de las menciones del lugar Wakhab'nal, que era posiblemente una forma antigua del nombre de Chichén Itzá, y es usada en forma cercana en el Chilam Balam como Uachabnal (6 Matorrales) y Uucyabnal (7 Matorrales). El topónimo Wakhab'nal en el Caracol está precedido por otros dos nombres de lugar: Tzaj y Tz'iknal. Puede argumentarse que Tzaj corresponde al complejo del Caracol, mientras Tz'iknal se referiría a la torre circular del Caracol (el Templo de Ehecatl-Quetzalcoatl), quizás como una referencia astronómica (Voss 2001), mientras Chichén Itzá en su conjunto se denominaría Wakhab'nal, cuya traducción más cercana sería "Los 6 Bosques de Matorrales" (Boot 2005:137, 292 y346) durante el siglo noveno dC.

En Chichén Itzá el topónimo ya-b'a-? o WAK-HAB?/?-NAL-la/la que se menciona en el área del Caracol, el Cuadrante 7B y el Templo de los Cuatro Dinteles estaba especialmente ligado a tres personajes: K'ak' Upakal, K'inil Kopol y Ak'e Tok'. En el texto frontal del Dintel 2 de la última estructura, la residencia de K'inil Kopol es denominada ya-b'a-?, que es similar a las voces de las fuentes coloniales yab'(an) o hab'(an), traducido como "matorral" (Boot 2005:343).

La Figura 4 muestra un mapa de los edificios conocidos de estilo "Maya Puuc", usando como referencia geográfica los cenotes y rejolladas más prominentes. Nótese que las construcciones de estilo "Tolteca" del Postclásico temprano no se presentan en el mapa, dándonos una idea más clara de la configuración de la ciudad en tiempos de K'ak' Upakal. La noción más aparente que revela este plano es la disposiciónde la ciudad en un eje vertical, norte-sur, a lo largo del cual se distribuyen los principales grupos. En segundo lugar, observando la extensión total del asentamiento en este tiempo notamos que la mayoría de las construcciones se sitúan al sur del Cenote Xtolok. Pocas construcciones de esta época se encuentran en las cercanías del Cenote Sagrado, con excepción de dos fases constructivas de la Gran Nivelación (Schmidt y Pérez de Heredia 2005). Sin embargo, la cerámica del Clásico terminal recuperada del Cenote Sagrado (Pérez de Heredia 2010) muestra claramente que este pozo estaba definitivamente integrado en el funcionamiento de la ciudad como fuente de agua y quizás también como un espacio ritual y sagrado ya desde esa época, aunque no en la medida en que lo sería durante el Postclásico temprano y periodos posteriores.

Para poder entender mejor el patrón de asentamiento de la ciudad debemos considerar la relación entre los grupos arquitectónicos del Clásico terminal y las mejores parcelas, usualmente localizadas donde están las rejolladas. Es relevante mencionar el nombre del moderno pueblo de Xcalacoop, situado a ocho kilómetros al sureste de Chichén Itzá, que significa "entre dos rejolladas", siendo este nombre la descripción exacta de la localización del pueblo entre dos profundas depresiones del terreno. El mapa de la Figura 4 muestra disposiciones similares en Chichén Itzá, donde varios grupos arquitectónicos fueron situados muy cerca o entre dos grandes rejolladas. Estos conjuntos arquitectónicos ordenados así pueden además asociarse con 


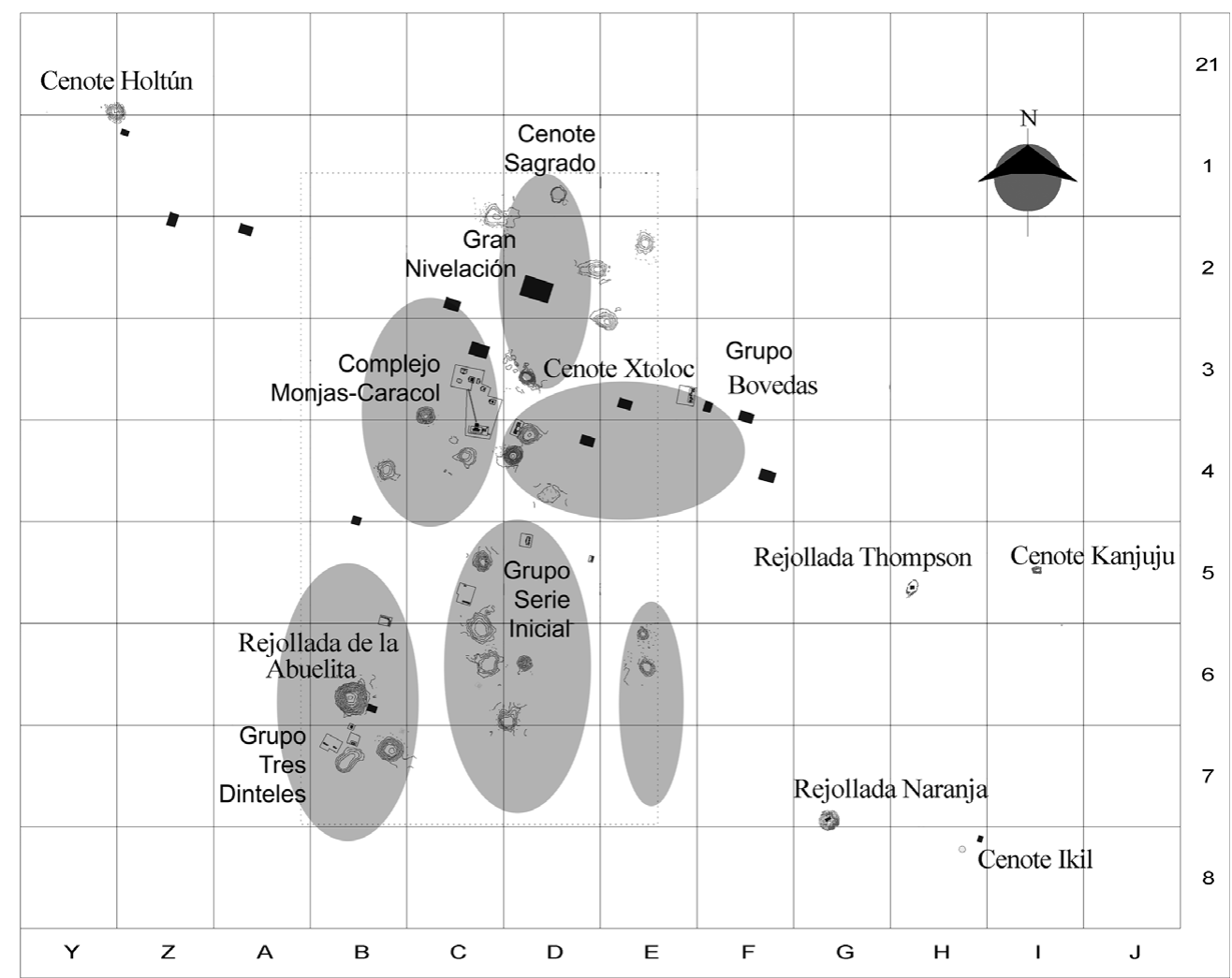

Figura 4. Cenotes, rejolladas y grupos arquitectónicos de Chichen Itzá a fines del siglo noveno. Los rectángulos en gris oscuro muestran la distribución de los contextos arquitectónicos contenientes cerámica del complejo Cehpech. Los óvalos en gris claro muestran las posibles divisiones de la ciudad. La extensión de cada unidad es arbitraria y tentativa (dibujo de Travis Nygard).

grupos familiares presentes en las inscripciones, resultando en una distribución territorial de los principales linajes.

Las fuentes coloniales establecen claramente que durante el período Postclásico las mejores rejolladas eran posesión de las familias nobles, y éste puede haber sido el caso desde el Preclásico en el norte de Yucatán. Basándonos en las inscripciones podemos entender que los Kokom se asociaban con el par de rejolladas del noreste y el Akabdzib, mientras el grupo de K'inil Kopol se asociaba con el xcalacoop del suroeste, asociado con la Rejollada de la Abuelita, donde varios conjuntos monumentales eran posesión de K'inil Kopol. Mientras tanto, K'ak' Upakal poseía el complejo de MonjasCasa Colorada en el noroeste, donde se situaba el asiento de poder real, también asociado a un par de rejolladas. Es posible, aunque la evidencia es tenue, que el grupo Chuk pueda haber estado vinculado al xcalacoop que limita el grupo de la Estructuras 6E3 y 6E1, en el sector sureste de la ciudad. Finalmente, el Grupo de la Serie Inicial, construido entre dos rejolladas, podría haber estado asociado con el culto de los ancestros de la casa real, y controlado conjuntamente por los tres hermanos.

Un importante conjunto escapa al arreglo estricto de xcalacoop: el Cenote Xtolok, una fuente de agua permanente fácilmente accesible, conectado por proximidad con el edificio del Caracol-Tz'iknal en Clasico Terminal sin duda, y que proponemos denominar como el área Tsaj. Otra excepción a este patrón es el gran conjunto de edificios alrededor del Castillo Viejo, que no se asocia con rejollada ni cenote alguno. Sin embargo, esta anomalía podría explicarse desde la cronología, dado que el único edificio 
con inscripciones de ese grupo, la pirámide del Castillo Viejo (Schmidt et al. 2008), postdata el reinado de K'ahk' Upakal. Es notable, además, la ausencia de pirámides durante este período. El templo principal de la ciudad era sin duda el Caracol, el Templo de Ehecatl-Quetzalcoatl, mientras el culto de los ancestros del linaje gobernante Kanek' se daba en el Templo de los Falos del grupo de la Serie Inicial. Asimismo, es de notar que el patrón de ocupación es comparable al de Utatlán, con Kanek' en el oeste, el templo de Quetzalcoatl en el centro y el juez Kokom en el este.

\section{Conclusión}

Hemos mostrado en este artículo que la organización política de Chichén Itzá durante el Clásico terminal muestra características que la distancian del modelo de realeza del Clásico tardío. Algunas de estas, como el patrón de gobierno por dos triadas propuesto para este período, son heraldos de posteriores modelos políticos del período Postclásico, registrados para el área de los lagos del Petén o las tierras altas de Guatemala. Podríamos incluso decir que representa una de las más antiguas evidencias del sistema de organización política que ha sido denominada los "Zuyuanos" (López Austin y López Luján 2000). Acompañando a los cambios políticos se da también la introducción del culto de Quetzalcoatl, en su forma de Ehecatl, como en otros sitios del sur (Seibal, Ucanal, Jimbal). Esta misma mezcla de rasgos antiguos y novedosos es aparente en otras ciudades del Clásico terminal del norte peninsular (Pérez de Heredia y Bíró 2018; Ringle et al. 1998).

Desde hace un par de décadas algunos investigadores propusieron la teoría de que en el período Clásico tardío el número de nobles aumentó, los reyes autocráticos se vieron obligados a compartir el poder en mayor medida y comenzó a emerger una forma más descentralizada de gobierno (Fash 1991; Houston y Stuart 2001; Jackson 2013, 2016; Webster 1999, 2000, 2004). Varios epigrafistas han analizado la evidencia disponible y han concluido que este crecimiento de la nobleza tuvo un carácter regional, porque de los títulos examinados por ellos el $73 \%$ pertenece al área occidental y sólo el $9 \%$ se encuentra en la región central, predominando los textos en vasijas cerámicas (Bíró 2012; Jackson 2013; Zender 2004:390-391). Por otra parte, en aquellas áreas donde creció el número de los nobles ya existían antecedentes en el período Clásico temprano, sugiriendo entonces que de alguna manera este fenómeno estaba relacionado con la posición periférica de la zona (Bíró 2011:293-295). Mientras tanto, solamente ocurre un ejemplo (Toniná) en las ciudades occidentales en el que un personaje que no pertenecía a la familia real utiliza el adjetivo $k^{\prime} u h u l$ (divino).

En cuanto al término que hemos empleado de "compartir el poder" es necesario hacer algunas precisiones. En contraste con los sitios del Clásico tardío del sur, en Chichén Itza cada uno de los personajes más prominentes de la nobleza utiliza el adjetivo k'uhul, y los títulos de los nobles eran únicos y exclusivos de la ciudad, a excepción de los que están asociados con las ceremonias del fuego y el propio título de rey divino. Además, de acuerdo a las inscripciones, casi todos los nobles realizaron ritos en común. Es evidente que estos datos permiten interpretaciones diversas, pero en nuestra opinión el cambio sustantivo se da en la calidad de los títulos, que corresponde con un proceso de pérdida de rango de los títulos en el período Clásico terminal (Bíró 2012). Es interesante que en Chichén Itzá el k'uhul ajaw agregaba a éste otra serie de títulos para distinguirse más de la nobleza, mientras anteriormente era suficiente el título de rey divino para lograrlo. Este panorama parece sugerir cierta competencia entre el rey y los nobles. Hay que añadir que no hay ninguna palabra en los textos que muestre alguna relación subordinada entre los miembros de la cumbre del reino, lo que sugiere de nuevo una relación menos jerárquica que en el periodo Clásico.

En resumen, la distribución de grupos arquitectónicos del Clásico terminal en Chichén Itzá muestra una preferencia por parejas de rejolladas o xcalacoop. Cinco conjuntos encajan en este patrón, a los que habría que sumar el caso particular del Caracol que está asociado al Cenote Xtolok, para alcanzar un total de seis particiones. Esto podría reflejar el patrón expresado en el antiguo nombre de Chichén Itzá como Wakhab'nal, traducido 
como "Seis - Monte/Grande - Matorral/Lugar de Hierbas/Milpas" (Boot en Schele y Mathews [1998:355, nota 7]). Aquí es relevante la opinión de Boot (2005:137) de que Wakhab'nal (6 Matorrales) era del nombre del "Chichén Viejo", y de que la ciudad recibiría el nombre Uucyabnal (7 Matorrales) cuando los edificios de la Gran Nivelación fueron construidos en el nuevo estilo "Tolteca".

Por análisis epigráfico es posible asignar estas particiones a algunas de las familias prominentes de la época, con K'ak' Upakal en el noroeste (Monjas-Casa Colorada), el linaje Kokom en el noreste (Akabdzib), Kopol en el suroeste y quizás los Chuk en el sureste. La particular configuración política de Chichén Itzá puede haber sido también consecuencia de luchas faccionales, que han sido detectadas entre los linajes reales de importantes ciudades del Clásico. Mientras se daba continuidad a la sucesión padre-hijo al trono Kanek', la organización de la Casa Real incluía al hermano del gobernante, quien se beneficiaba con el usufructo de un importante sector de ricas tierras. Es posible suponer que el sistema permitía que, en caso de falta de heredero, la sucesión pasase al segundo de la casa real, y quizás esto es lo que haya sucedido a la muerte de K'ak' Upakal en Chichén Itzá, dado que no conocemos a ningún sucesor directo. Es interesante recordar aquí el aserto de Landa de que los tres hermanos (por castos) no tuvieron descendencia, y que tras el caos que siguió a su muerte, llegó Kukulkan a pacificar la tierra.

Si bien la organización de la Casa Real (que se identifica con el sur de la ciudad) estaba asegurada por un trío de miembros cercanos de una sola familia, la organización de la polis, que se sitúa en el norte de la ciudad, se dividió en tres oficinas ejecutivas. El gobernante supremo era también el líder de este trío, dado que es el general del ejército, el Kanek', la serpiente emplumada de la guerra. Segundo en importancia ejecutiva aparece Yajawal Cho, el k'uhul kokom, quien fungía como principal consejero, oidor real y también brazo armado del rey, ocupando el Akabdzib en el noreste y sus rejolladas asociadas. El tercer miembro de esta triada es el sacerdote de Quetzalcoatl, vocero e intérprete de la divinidad, ocupando el Caracol. Esto sugiere una incipiente institución sacerdotal, lo que de probarse constituiría otra modificación importante del nuevo sistema político.

Agradecimientos. A los maestros que siguen leyendo y editando nuestros trabajos nuestro agradecimiento más sincero: Peter J. Schmidt, Sylviane Boucher, Peter Mathews, Geoffrey Braswell, Barbara MacLeod, Guido Krempel y Carlos Pallán.

Declaración de Disponibilidad de Datos. No se presentan datos originales en este artículo.

\section{Referencias Citadas}

Andrews, Anthony P. y Fernando Robles Castellanos

1985 Chichén Itzá and Cobá: An Itzá-Maya Standoff in early Postclassic Yucatan. En The Lowland Maya Postclassic, editado por Arlen F. Chase y Prudence M. Rice, pp. 62-72. University of Texas Press, Austin.

Andrews, E. Wyllys V y Jeremy A. Sabloff

1986 Classic to Postclassic: A Summary Discussion. En Late Lowland Maya Civilization: Classic to Postclassic, editado por Jeremy A. Sabloff y E. Wyllys Andrews V, pp. 433-456. University of New Mexico Press, Albuquerque.

Andrews, Anthony P., E. Wyllys Andrews V y Fernando Robles Castellanos

2003 The Northern Maya Collapse and its Aftermath. Ancient Mesoamerica 14:151-156.

Barrera Vásquez, Alfredo y Silvia Rendón

1948 El Libro de los Libros de Chilam Balam. Fondo de Cultura Económica, México, D.F.

Baudez, Claude-François y Nicolas Latsanopoulos

2010 Political Structure, Military Training, and Ideology at Chichén Itza. Ancient Mesoamerica 21: $1-20$.

Bíró, Péter

2011 The Classic Maya Western Region: A History. British Archaeological Reports International Series S2308. Hadrian Books, Oxford, Inglaterra.

2012 Politics in the Western Maya Region (III): The Royal and the Non-Royal Elite. Estudios de Cultura Maya 40:79-96.

Bíró, Péter y Eduardo Pérez de Heredia

2016 The Caracol Disk of Chichén Itzá (929-932 CE): Some Thoughts on Epigraphy and Iconography. Estudios de Cultura Maya 48:129-162.

Boot, Erik

2005 Continuity and Change in Text and Image at Chichén Itzá, Yucatan, Mexico: A Study of the Inscriptions, Iconography, and Architecture at a Late Classic to Early Postclassic Maya Site. CNWS Publications, Leiden.

Brainerd, George W.

1958 The Archaeological Ceramics of Yucatan. Anthropological Records 19. University of California Press, Berkeley y Los Angeles.

Carmack, Robert M.

1973 Quichéan Civilization: The Ethnohistoric, Ethnographic and Archaeological Sources. University of California Press, Berkeley y Los Angeles. 
1981 The Quiché Mayas of Utatlán: The Evolution of a Highland Guatemala Kingdom. University of Oklahoma Press, Norman.

Cobos Palma, Rafael

2004 Chichén Itzá: Settlement and Hegemony during the Terminal Classic Period. En The Terminal Classic in the Maya Lowlands: Collapse, Transition, and Transformation, editado por Arthur A. Demarest, Prudence M. Rice y Don S. Rice, pp. 517-544. University Press of Colorado, Boulder.

Davoust, Michel

1977 Etude epigraphique 2: les chefs Mayas de Chichén Itzá et les glyphs de filiation. Angers, Francia.

De la Garza, Mercedes, Ana Luisa Izquierdo, Ma. Del Carmen León Caźares y Tolita Figueroa

1983 Relaciones histórico-geográficas de la gobernación de Yucatán, Vol. I-II. Universidad Nacional Autónoma de México, México, D.F.

Edmonson, Munro S.

1982 The Ancient Future of the Itzá: The Book of Chilam Balam of Tizimin. University of Texas Press, Austin.

1986 Heaven Born Merida and its Destiny. The Book of Chilam Balam of Chumayel. University of Texas, Austin.

Fash, William

1991 Scribes, Warriors and Kings: The City of Copán and the Ancient Maya. Thames and Hudson, New York.

Fox, John W.

1989 On the Rise and Fall of Tuláns and the Maya Segmentary States. American Anthropologist 91(83):656681.

Gómez Pompa, Arturo

1990 The Sacred Cacao Groves of the Maya. Latin American Antiquity 1:247-257.

González de la Mata, Rocío

2006 Agua, agricultura y mitos: El caso de tres rejolladas de Chichén Itzá. En XIX Simposio de Investigaciones Arqueológicas en Guatemala, 2005, editado por Juan Pedro Laporte, Bárbara Arroyo y Héctor E. Mejía, pp.305-318. Museo Nacional de Arqueología y Etnología, Guatemala.

Grube, Nikolai

1994 Hieroglyphic Sources for the History of Northwest Yucatan. En Hidden Among the Hills: Maya Archaeology of the Northwest Yucatan Peninsula, editado por Hanns J. Prem, pp. 316-358. Acta Mesoamericana 7. Verlag von Flemming, Möckmühl.

2003 Hieroglyphic Inscriptions from Northwest Yucatan: An Update of Recent Research. En Escondido en la selva: Arqueologia en el norte de Yucatan, editado por Hanns J. Prem, pp. 339-370. Instituto Nacional de Antropología e Historia, México, D.F.

Grube, Nikolai y Ruth Krochock

2007 Reading between the Lines: Hieroglyphic Texts from Chichén Itzá and its Neighbours. En Twin Tollans: Chichén Itzá, Tula, and the Epiclassic to Early Postclassic Mesoamerican World, editado por Jeff Karl Kowalski y Cynthia Kristan-Graham, pp. 205-250. Dumbarton Oaks Research Library and Collection, Washington, DC.

Grube, Nikolai, Alfonso Lacadena y Simon Martin

2003 Chichén Itzá and Ek Balam. Notebook for the XXVIIth Maya Hieroglyphic Forum at Texas, March 2003. Department of Art History, University of Texas, Austin.
Houston, Stephen D. y David Stuart

2001 Peopling the Classic Maya Court. En Royal Courts of the Ancient Maya, vol. 1, editado por Takeshi Inomata y Stephen D. Houston, pp. 54-83. Westview Press, Boulder.

Hull, Kerry M. y Michael D. Carrasco ( editores)

2014 Parallel Worlds: Genre, Discourse, and Poetics in Contemporary, Colonial, and Classic Maya Literature. University Press of Colorado, Boulder.

Jackson, Sarah

2013 Politics of the Maya Court: Hierarchy and Change in the Late Classic Period. University of Oklahoma, Norman.

2016 Governing Polities: Royal Court and the Written Landscape of the Late Classic Maya Politics. En Classic Maya Polities of the Southern Lowlands: Integration, Interaction, Dissolution, editado por Damien B. Marken y James L. Fitzsimmons, pp. 243-262. University Press of Colorado, Boulder.

Johnson, Scott A.J.

2015 The roots of Sotuta: Dzitas Slate as a Yucatecan Tradition. Ancient Mesoamerica 26:113-126.

Jones, Grant D.

1998 The Conquest of the Last Kingdom. Stanford University Press, Stanford, California.

Kelley, David H.

1968 Kakupacal and the Itzás. Estudios de Cultura Maya 7:255-268.

Kepecs, Susan y Sylviane Boucher

1996 The Prehispanic Cultivation of Rejolladas and Stone Lands: New Evidence from Northeast Yucatan. En The Managed Mosaic: Ancient Maya Agriculture and Resource Use, editado por Scott L. Fedick, pp. 69-91. University of Utah Press, Salt Lake City.

Kirchhoff, Paul

1955 Quetzlcoatl, Huemac y el fin de Tula. Cuadernos Americanos LXXXIV:163-196.

Krochock, Ruth J.

1988 A New Interpretation of the Inscriptions on the Temple of the Hieroglyphic Jambs, Chichén Itzá. Texas Notes on Precolumbian Art, Writing, and Culture 79. University of Texas at Austin, Center of the History and Art of Ancient American Culture, Austin.

1989 Hieroglyphic Inscriptions at Chichén Itzá, Yucatan, Mexico: The Temples of the Initial Series, the One Lintel, the Three Lintels, and the Four Lintels. Research Reports on Ancient Maya Writing, 23. Center for Maya Research, Washington, DC.

Kristan-Graham, Cynthia y Jeff Karl Kowalski

2007 Chichén Itzá, Tula, and Tollan: Changing Perspectives on a Recurring Problem in Mesoamerican Archaeology and Art History. En Twin Tollans: Chichén Itzá, Tula, and the Epiclassic to Early Postclassic Mesoamerican World, editado por Jeff Karl Kowalski y Cynthia Kristan-Graham, pp. 13-84. Dumbarton Oaks Research Library and Collection, Washington, DC.

Lincoln, Charles E.

1990 Ethnicity and Social Organization at Chichén Itzá, Yucatan, Mexico. Tesis doctoral, Department of Anthropology, Harvard University, Cambridge, Massachusetts.

López Austin, Alfredo y Leonardo López Luján

2000 The Myth and Reality of Zuyuá: The Feathered Serpent and Mesoamerican Transformations from the Classic to the Posclassic. En Mesoamerica's Classic Heritage: From Teotihuacan to the Aztecs, editado por 
Davíd Carrasco, Lindsay Jones y Scott Sessions, pp. 21-84. University Press of Colorado, Boulder.

MacLeod, Barbara

2004 A World in a Grain of Sand: Transitive Perfect Verbs in the Classic Maya Script. En The Linguistics of Maya Writing, editado por Søren Wichmann, pp. 291-326. University of Utah Press, Salt Lake City.

Morley, Sylvanus G.

1946 Ancient Maya. Stanford University Press, Stanford. Paulinyi, Zoltan

1981 Capitals in Pre-Aztec Central Mexico. Acta Orientalia Academiae Scientiarum Hungaricae XXXV(23):315-350.

Pérez de Heredia, Eduardo J.

2010 Ceramic Contexts and Chronology at Chichén Itzá, Yucatan, Mexico. Tesis doctoral, La Trobe University, Melbourne, Australia.

Pérez de Heredia, Eduardo J. y Péter Bíró

2017 Ceramics and Epigraphy: Coba and the History of Northern Yucatán AD 700-830. Manuscrito inédito. https://www.academia.edu/21907081/Ceramics_and_ Epigraphy_Coba_and_the_history_of_Northern_ Yucatán_AD_700-830, accedido el 14 de junio de 2016.

2018 K'ak' Upakal K'inich K'awil and the Lords of the Fire: Chichén Itzá during the Ninth Century. En Landscapes of the Itzá: Archaeology and Art History at Chichén Itzá and Neighboring Sites in Yucatan, Mexico, editado por Linnea Wren, Cynthia KristanGraham, Kaylee Spencer, y Travis Nygard, pp. 65-108. University Press of Florida, Gainesville.

Plank, Shannon

2003 Maya Dwellings in Hieroglyphs and Archaeology: An Integrative Approach to Ancient Architecture and Spatial Cognition. Tesis doctoral, Boston University, Boston.

Ringle, William

2004 On the Political Organiztion of Chichén Itzá. Ancient Mesoamerica 15:167-218.

2009 The Art of War: Imagery of the Upper Temple of the Jaguar, Chichén Itzá. Ancient Mesoamerica 20: $14-44$.

2017 Debating Chichén Itza. Ancient Mesoamerica 28:119-136.

Ringle, William M., Tomás Gallareta Negrón y George J. Bey III

1998 The Return of Quetzalcoatl: Evidence for the Spread of a World Religion during the Epiclassic Period. Ancient Mesoamerica 9:183-232.

Roys, Ralph L.

1962 Literary Sources for the History of Mayapán. En Mayapán, Yucatan, Mexico, editado por Henry E. D. Pollock, Ralph L. Roys, Tatiana Proskouriakoff y A. Ledyard Smith, pp. 25-86. Publication 619, Carnegie Institution of Washington, Washington, DC.

Ruppert, Karl

1952 Chichen Itza: Architectural Notes and Plans. Publication 595, Carnegie Institution of Washington, Washington, DC.

Sabloff, Jeremy A. y E. Wyllys Andrews V (editores)

1986 Late Lowlands Maya Civilization: Classic to Postclassic. University of New Mexico Press, Albuquerque.

Schele, Linda y David Freidel

1990 A Forest of Kings: The Untold Story of the Ancient Maya. William Morrow, New York.
Schele, Linda y Peter Mathews

1998 The Code of Kings: The Language of Seven Sacred Maya Temples and Tombs. Scribner, New York.

Schmidt, Peter J.

2003 Siete años entre los Itzá: Nuevas excavaciones en Chichén Itzá y sus resultados. En Escondido en la selva: Arqueologia en el norte de Yucatan, editado por Hanns J. Prem, pp. 53-64. Instituto Nacional de Antropología e Historia, México, D.F.

2007 Birds, Ceramics, and Cacao: New Excavations at Chichén Itzá, Yucatan. En Twin Tollans: Chichén Itzá, Tula and the Epiclassic to Early Postclassic Mesoamerican World, editado por Jeff K. Kowalski y Cynthia Kristan-Graham, pp. 151-203. Dumbarton Oaks, Washington, DC.

Schmidt, Peter J. y Eduardo Pérez de Heredia Puente

2006 Fases de construcción de la Gran Nivelación de Chichen Itzá. Ponencia presentada en el XVI Encuentro Internacional "Los Invetigadores de la Cultura Maya," Campeche.

Schmidt, Peter, David Stuart y Bruce Love

2008 Inscriptions and Iconography of Castillo Viejo, Chichén Itzá. PARI Journal IX(2):1-17.

Smith, Robert E.

1971 The Pottery of Mayapan, Including Studies of Ceramic Material from Uxmal, Kabah, and Chichen Itza. Papers of the Peabody Museum of Archaeology and Ethnology Vol. 66, Harvard University, Cambridge, Massachusetts.

Thompson, J. Eric S.

1937 A New System for Deciphering Yucatecan Dates with Special Reference to Chichén Itzá. Publication 438, Carnegie Institution of Washington, Washington, DC.

1954 The Rise and Fall of Maya Civilization. Civilizations of the American Indian Series 39. University of Oklahoma Press, Norman.

Tozzer, Alfred M.

1957 Chichén Itzá and Its Cenote of Sacrifice: A Comparative Study of Contemporaneous Maya and Toltec. Memoirs of the Peabody Museum of American Archaeology and Ethnology Vol 11-12, Harvard University, Cambridge, Massachusetts.

Volta, Beniamino y Geoffrey E. Braswell

2014 Alternative Narratives and Missing Data: Refining the Chronology of Chichén Itzá. En The Maya and their Central American Neighbors: Settlement Patterns, Architecture, Hieroglyphic Texts, and Ceramics, editado por Geoffrey E. Braswell, pp. 356-403, Routledge, Londres y Nueva York.

Voss, Alexander

2001 Los Itzáes en Chichén Itzá: Los datos epigráficos. En Los Investigadores de la Cultura Maya 9, Tomo I, pp. 152-173, Universidad Autónoma de Campeche, Campeche.

Voss, Alexander y Jürgen Kremer

2000 K'ak'-u-pakal, Hun-pik-tok' and the Kokom: The Political Organization of Chichén Itzá. En The Sacred and the Profane: Architecture and Identity in the Maya Lowlands, editado por Pierre R. Colas, Kai Delvendahl, Marcus Kuhnert y Annette Schubart, pp. 149-182. Acta Mesoamericana, 10, Verlag Anton Saurwein, Markt Schwaben.

Webster, David

1999 Warfare and Status Rivalry: Lowland Maya and Polynesian Comparisons. En Archaic States, editado por 
Gary M. Feinman y Joyce Marcus, pp. 311-351. School of American Research Press, Santa Fe.

2000 Not so Peaceful Civilization: a Review of Maya War. Journal of World Prehistory 4(1):65-119.

2004 Political Ecology, Political Economy, and the Culture History of Resource Management at Copán. En Copan: The History of an Ancient Maya Kingdom, editado por E. Wyllys Andrews V y William L. Fash, pp. 33-72. School of American Research Press, Santa Fe.

Wren, Linnea y Peter Schmidt

1991 Elite Interaction during the Terminal Classic Period: New Evidence from Chichén Itzá. En Classic Maya Political History: Epigraphic and Archaeological Evidence, editado por T. Patrick Culbert, pp.199-225. Cambridge University Press, Cambridge.
Zender, Marc

2004 A Study of Classic Maya Priesthood. Tesis de doctorado, Department of Archaeology, University of Calgary, Calgary.

\section{Nota}

1. Al usar el término estilo arquitectónico Tolteca en este artículo pretendemos enfatizar el parecido de los edificios del Postclásico temprano con aquellos de Tula, Hidalgo, en contraste con el estilo arquitectónico Maya-Puuc del Clásico terminal, sin pretender atribuir filiaciones étnicas en ninguno de los dos casos. Otros autores prefieren el termino Estilo Internacional para referirse al fenómeno arquitectónico Tolteca.

Submitted February 17, 2017; Revised May 10, 2017; Accepted November 29, 2017 